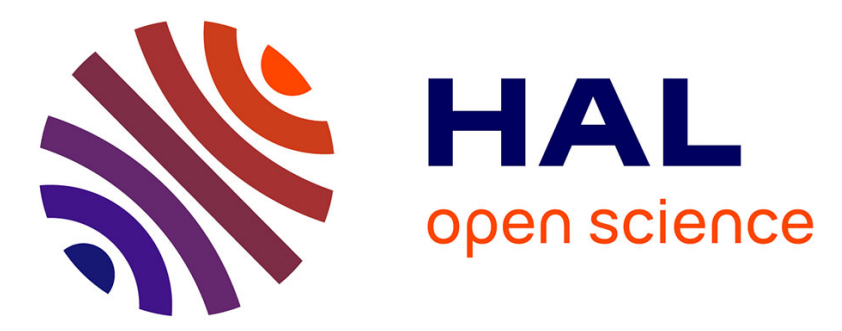

\title{
A framework for backbone experimental tracking : piezoelectric actuators, stop-sine signal and kalman filtering
}

\author{
Nicolas Peyret, Jean-Luc Dion, Gael Chevallier
}

\section{- To cite this version:}

Nicolas Peyret, Jean-Luc Dion, Gael Chevallier. A framework for backbone experimental tracking: piezoelectric actuators, stop-sine signal and kalman filtering. Mechanical Systems and Signal Processing, 2016, 78, pp.28 - 42. 10.1016/j.ymssp.2015.09.020 . hal-01446386

\author{
HAL Id: hal-01446386 \\ https://hal.science/hal-01446386
}

Submitted on 25 Jan 2017

HAL is a multi-disciplinary open access archive for the deposit and dissemination of scientific research documents, whether they are published or not. The documents may come from teaching and research institutions in France or abroad, or from public or private research centers.
L'archive ouverte pluridisciplinaire HAL, est destinée au dépôt et à la diffusion de documents scientifiques de niveau recherche, publiés ou non, émanant des établissements d'enseignement et de recherche français ou étrangers, des laboratoires publics ou privés. 


\title{
A framework for backbone experimental tracking : piezoelectric actuators, stop-sine signal and kalman filtering Nicolas PEYRET ${ }^{\mathrm{a}}$, Jean-Luc DION ${ }^{\mathrm{a}}$, Gael CHEVALLIER ${ }^{\mathrm{b}, *}$ \\ ${ }^{a}$ Laboratoire QUARTZ EA7393 SUPMECA PARIS - 3 rue Fernand Hainaut - F-93407 SAINT OUEN, \\ France \\ ${ }^{b}$ FEMTO-ST Institute - UMR 6174, CNRS-UFC-ENSMM-UTBM, 24, chemin de l'Epitaphe, F-25000 \\ Besancon, France
}

\begin{abstract}
This paper deals with the use of piezoelectric patches for nonlinear dynamic identification. The patches are glued on the structure to identify amplitude-dependent damping and natural frequency; their positions are defined in order to perform the excitation concentrated on the first bending mode. Their locations on the structure allow to perform "stop sines" tests, as, unlike electrodynamic shakers, piezos are embedded on structures and do not modify the studied structure after the excitation signal is switched off. Although, despite the piezo and the stop-sine, the signal is still modulated by other frequency components or polluted by random signals, a post processing with the extended Kalman Filter allows a very good determination of the modal damping and the natural frequency, especially when they depends on the free vibration amplitude.
\end{abstract}

Keywords: Piezoelectric excitation, Non Linear Vibrations, Kalman Filter

\section{Introduction}

In order to compute the vibration levels of built-up structures, the prediction of damping remains a great challenge. Simulation tools, Computer Aided Design and Finite Element Method, are used to predict both inertia and stiffness with a pretty good accuracy, but damping is often badly estimated. As a consequence, the vibration levels

\footnotetext{
*Corresponding author. E-mail adress: gael.chevallier@univ-fcomte.fr, Tel.: +33 662696523

${ }^{1}$ E-mail adress: nicolas.peyret@ supmeca.fr

${ }^{2}$ E-mail adress: jean-luc.dion@supmeca.fr
} 
are also wrongly predicted. The damping might be induced through several common ways such as viscoelastic materials, pressure loss in fluids or solid friction. The latter remains badly modeled, whereas the joints, such as welded points, bolted joints or rivets, are widely used to link the parts of the mechanisms and the structures.

\subsection{State of the art}

Among all the studies that focus on friction-damping, it is commonplace to separate the works that highlight energy dissipations coming from macro-sliding, and micro-sliding. In the first category, the damping is due to localized friction points, see Berthillier et al.[1] or Poudou et al. [2] for instance. In this case, simulations and tests are easy to perform because the contact region is generally localized and the slipping occurs all over the contact area. In the second case, the damping comes from partial sliding between the parts. Thus the sliding region is generally badly known and the motion is governed by stick-slip waves between the parts. It was shown experimentally by many authors (Goodman et al. [3], Beards et al.[4], Pian [5], Ungar [6]) that, in turn, the damping is strongly dependent to the vibration amplitude. This is due to the pressure and the shear stress variations. This leads to difficulties in modelling this case, see Festjens et al. [7] and Caignot et al. [8]. In simulations, geometrical defects and loading trajectories have to be carefully taken into account. To avoid this difficulty, it is also possible to measure and identify meta-models, see Festjens et al. [9], on specifically designed test-benchs. Several testing devices have been designed in order to achieve this goal Fig.1. The advantages of most of the experimental setups, according to their design properties and their experimental process, were exposed in Dion et al. [10]. Special attention has been payed to the coupling between tangential and normal loads in the joints because this coupling makes the experiments quite hard to perform, as the limit of sliding is depending on the normal load dynamics.

In previous works, Peyret et al. [22, 10] proposed to study the Energy Losses (EL) on a new benchmark, Fig.1.j. This new academic assembly test was introduced because it was experimentally difficult to ensure constant normal stress in the previous benchmarks such as those suggested by Goodman et al. [3] and Metherel et al. [12], see Fig.1.b and Fig.1.f. Peyret's device is constituted of three parts linked together by 

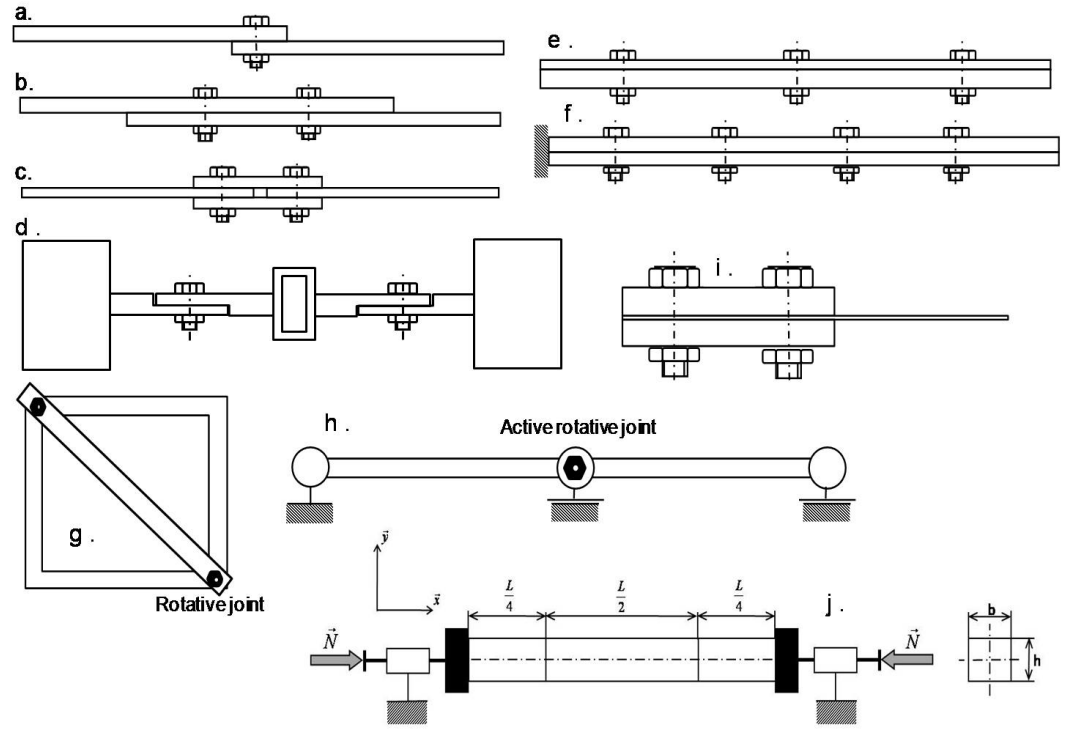

Figure 1: Exemples of testing devices develloped to highlight friction induced damping in joints. a. Beam assembly with a single bolted joint from Ahmadian et al. [11]b. Beam assembly with two bolted joints from Metherell et al [12] or Esteban et al. [13]-c. Beam assembly symetrically screwed with two bolted joints from Song et al. [14]- d. structure with two blocks, one spring and beam assembly with two bolted joint and with special lap joint geometry from Goyder et al [15] - e. beam assembly with distributed bolted joints in free conditions from Heller et al $[16,17]-\mathrm{f}$. beam assembly with distributed bolted joints in clamped-free conditions from Goodman et al [3], Nanda et al.[18, 19]- g. Polyarticulated structure with rotative friction joints from Beards et al [4] - h. beam assembly with an active rotative joint from Gaul et al. [20]- i. cantilever quartz beam dedicated to the study of microsliding in the clamp from Nouira et al [21]- j. Clamp clamp cutted beam with pure microsliding in the interfaces from Peyret et al [22] 
two planes $\partial \Omega_{F}$. The right and left parts are clamped to the ground and the third part is excited thanks to piezoelectric patches glued in the middle of the part. The aim is to excite the device on its first bending mode. The main advantage of this device is, that, in the interfaces, on the first vibration mode, the bending-induced normal stresses are nil. Thus, this allows to measure pure-shear stress, and its effect on the damping of the first bending mode. This test bench is the one, we study in the present paper, but the framework, "piezos", stop-sine, "Kalman" might also be used for others specimens.

Each of these experimental configurations had been tested with specific excitation device and excitation signals. There are several experimental ways to highlight nonlinear effects such as amplitude-dependent natural frequencies and damping, which are very commonplace for friction dampers. Many works are based on steady-state analysis in order to build Frequency Response Functions (FRF), [11, 4, 13] to estimate the EL, through the frequency bandwidth or the quality factor of each modes. EL can also be estimated through quasi-static analysis $[11,12,3]$. In this case, the objective is to build energy ratios for various loading trajectories. This experimental procedure is very close to the procedure performed with Dynamic Mechanical Analyzer (DMA) for viscoelastic properties identification. Transient analysis can also be performed: the classical approach is to excite the structure with an impact hammer, [23]; A more original approach is to obtain free-decay response by disconnecting the sine-excitation device to the structure since a steady-state response very close to the modal response was obtained, [16], [14]. This procedure is called stop-sine in the present paper. This procedure allows to get the so-called backbones of the system; that is to say, the amplitude-dependence of the natural frequency and the modal damping. These characteristics are very useful because they belongs to the kernel of the system and they do not depend on the excitation signal. For all this reasons, stop-sine has been used in this paper. In order to apply such kind of signal, one has to be able to get, firstly a stationary harmonic motion close to a modal vibration, and secondly a free vibration motion collinear to the previous vibration mode. To get these two distinct motions without any displacement jump, the system must have the same vibration properties before and after the excitation stops. It is very difficult to cut the mechanical link between the structure and the classical excitation devices like vibrating tables or 
electromagnetic shakers. In fact, cutting the electrical link leads to residual electromagnetic forces. Thus, in this paper, we propose to embed piezoelectric patches on the structure in order to provide the excitation force. As they are embedded the impedance modification during the excitation stop remains negligible.

In order to post-process the tests and to identify the parameters of the specimen, several methods can be used. For steady-state tests, FRF and force-displacement maps allow to identify the damping using the frequency bandwidth around the resonnance frequency. To evaluate amplitude-dependent damping during transient tests, the logarithmic decrement can be used on time-signals. To evaluate amplitude-dependent frequencies and damping, time-frequency maps obtained with spectrogram or wavelet $[24,16,17,21]$ are commonly used. In this paper, we will compare Hilbert Transform and an Extended Kalman Filter to get instantaneous frequency and damping.

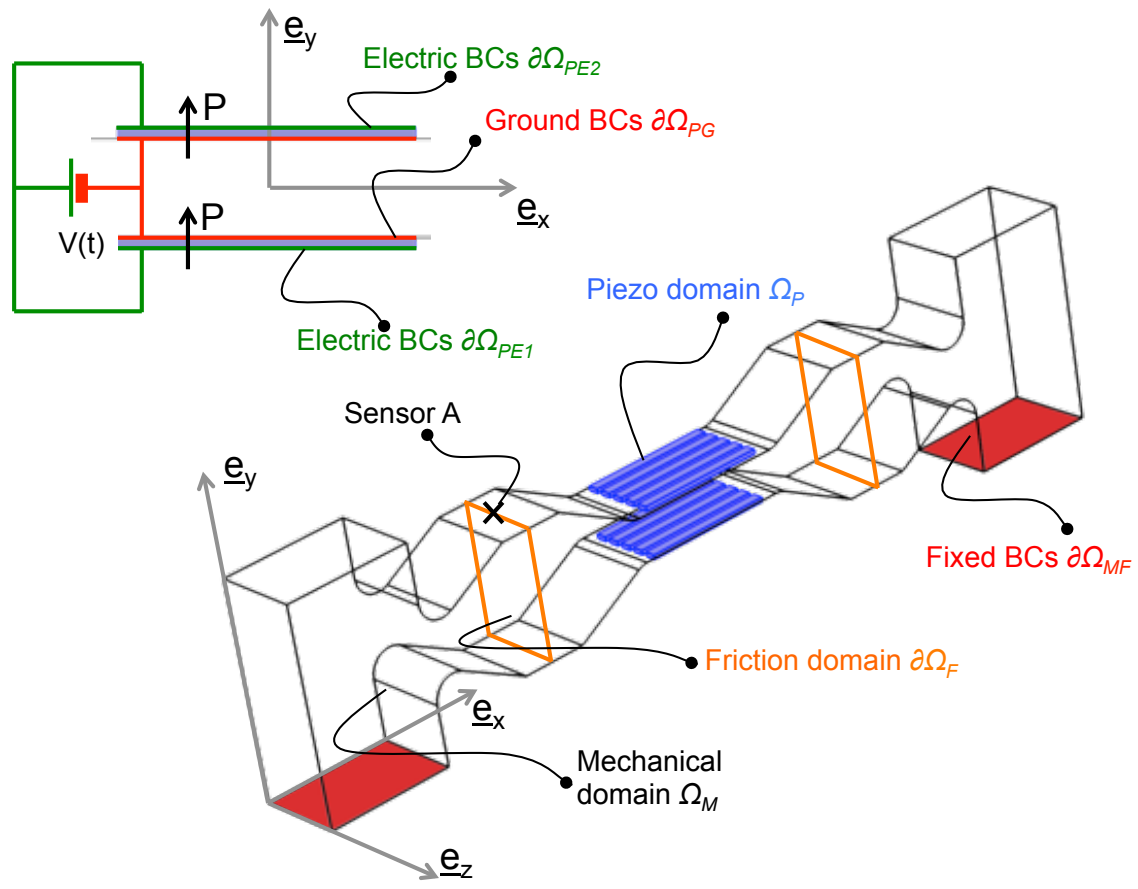

Figure 2: Schema of the test-bench. Definition of each subdomains and each boundary conditions 


\section{Piezoelectric Excitation Design}

The aim of this section is to justify the use of piezos for stop-sine tests. In order to measure the dependence of the first natural frequency and modal damping to the amplitude of vibrations, one expect to find the best positions and shapes for the actuators. To demonstrate the efficiency of such actuators, we assume that vibrations can be decomposed on the normal modes. In the case where non linearities come from partial sliding in the joints, this assumption is commonplace and has been justified by several authors such as Festjens et al. [9].

Firstly, the actuators $\Omega_{P}$ have to transmit as well as possible the axial stress to the structure $\Omega_{M}$ along the interface $\partial \Omega_{P G}$. As the actuators are glued on the main structure, this goal is achieved when the surface defects does not perturbate the stress path. In our case, the actuators are thin enough to be less sensitive to surface defects, see Figure 2.

Secondly, the positions of the actuators have to provide a great Electromechanical Coupling Coefficient (EMCC) $K_{i}$ for the first mode and lowers EMCC for other modes. The EMCC can be computed thanks to the "Open-Circuit" angular frequencies $\omega_{i}^{O C}$ and to the "Short-Circuit" frequencies $\omega_{i}^{S C}$, see eqn (1).

$$
K_{i}^{2}=\frac{\left(\omega_{i}^{O C}\right)^{2}-\left(\omega_{i}^{S C}\right)^{2}}{\left(\omega_{i}^{S C}\right)^{2}}
$$

These frequencies can be computed thanks to the resolution of elastodynamic equations enhanced with electrostatic equations and coupled with piezoelectric constitutive equations:

\begin{tabular}{l|l}
$\begin{array}{l}\text { Elastodynamics equations } \\
\text { on } \Omega_{M} \text { and } \Omega_{P}\end{array}$ & $\begin{array}{l}\text { Electrostatics equations and piezoelectric coupling } \\
\text { on } \Omega_{P}\end{array}$ \\
\hline \hline$T_{i j, j}=\rho \ddot{u}_{i}$ & $D_{i, i}=0$ \\
$S_{i j}=\frac{1}{2}\left(u_{i, j}+u_{i, j}\right)$ & $S_{i j}=s_{i j k l}^{E} T_{k l}+d_{k i j} E_{k}$ \\
$S_{i j}=s_{i j k l} T_{k l}$ & $D_{i}=d_{i k l} T_{k l}+\epsilon_{i k}^{T} E_{k}$ \\
& $E_{i}=\varphi_{, j}$
\end{tabular}

To determine the behavior of complex geometry structures, previous work suggested weak formulations for the development of numerical methods to approximate the be- 
havior of such structures. It is impossible to make a complete state of the art in this paper as the literature is abundant but Benjeddou's survey [25] gives an overview. In standard FE codes, the formulation with mechanical displacement and electric potential is often used:

$$
\begin{aligned}
& \int_{\Omega_{p}} c_{i j k l} \delta u_{i, j} u_{k, l} d V+\int_{\Omega_{p}} e_{k i j} \delta u_{i, j} \varphi_{, k} d V+\int_{\Omega_{p}} \rho \frac{\partial^{2} u_{i}}{\partial t^{2}} \delta u_{i} d V=0 \\
& -\int_{\Omega_{p}} e_{i k l} u_{k, l} \delta \varphi_{, i} d V+\int_{\Omega_{p}} \epsilon_{i k} \varphi_{, k} \delta \varphi_{, i} d V=0
\end{aligned}
$$

FE approximation leads to the following Differential Algebric Equation (DAE):

$$
\left[\begin{array}{ll}
M & \\
& 0
\end{array}\right]\left[\begin{array}{c}
\ddot{U} \\
\ddot{\Phi}
\end{array}\right]+\left[\begin{array}{cc}
K_{m} & K_{c} \\
-K_{c}^{T} & K_{e}
\end{array}\right]\left[\begin{array}{c}
U \\
\Phi
\end{array}\right]=\left[\begin{array}{l}
0 \\
0
\end{array}\right]
$$

Thanks to these equations and constraining the displacement field to satisfy the Dirichlet BCs on $\partial \Omega_{M F}$, the electric potential to satisfy the ground BCs $\partial \Omega_{P G}$ and the electric potential to be uniform on both electrodes $\partial \Omega_{P E 1}$ and $\partial \Omega_{P E 2}$, one can compute the "Open Circuit" frequencies.

$$
\left\{\begin{array}{l}
U=0 \text { on } \partial \Omega_{M F} \\
\Phi=0 \text { on } \partial \Omega_{P G} \\
\Phi=\Phi_{1} \text { on } \partial \Omega_{P E 1} \\
\Phi=\Phi_{2} \text { on } \partial \Omega_{P E 2}
\end{array}\right.
$$

Note that many users do not constrain the electric potential to be uniform on each electrodes. In this case, see Fig. 3, the EMCC may be wrong mainly for the uncoupled modes, see Table 1. This problem has been addressed in Chevallier et al. [26] and [27]. It is possible to understand "graphically", on Fig. 3, that the electrode makes the average of the potential all over surfaces of the patches; due to the anti-symetry of $\varphi$, the average potential is nil on Modes 2-5.

In order to compute the "Short Circuit" frequencies, one has just to add the following constraint to the previous equations:

$$
\Phi_{1}=\Phi_{2}
$$

Table 1 shows that, as expected, it is possible to design the actuators in order to get only one coupled mode among the five first modes. If the design is robust, one can expect to command only this mode. 


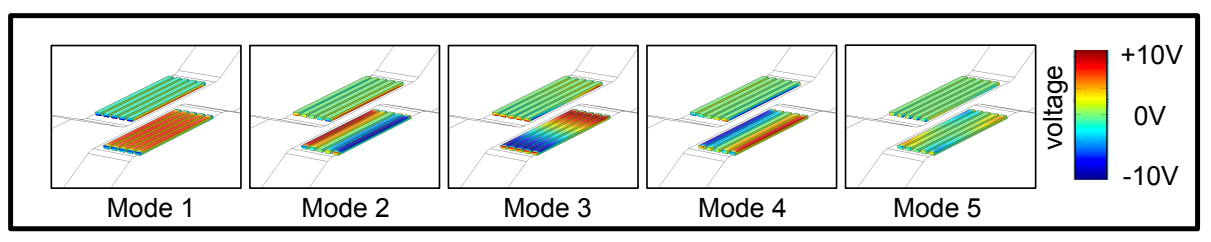

Figure 3: Patch voltages obtained with an eigenfrequency study with clamped mechanical BCs and 0V electrical BCs.

\begin{tabular}{c||c|c|c||c|c|c}
\hline Mode & $f_{i}^{O C} \mathrm{~Hz}$ & $f_{i}^{S C} \mathrm{~Hz}$ & $K_{i} \%$ & $f_{i}^{O C} \mathrm{~Hz}$ & $f_{i}^{S C} \mathrm{~Hz}$ & $K_{i} \%$ \\
\hline \hline 1 & 820.53 & 821.97 & 5.90 & 820.53 & 821.97 & 5.90 \\
2 & 898.76 & 899.17 & 3.03 & 898.76 & 898.76 & 0.00 \\
3 & 1852.52 & 1852.59 & 0.87 & 1852.52 & 1852.52 & 0.00 \\
4 & 2004.61 & 2006.15 & 3.92 & 2004.61 & 2004.61 & 0.00 \\
5 & 2029.17 & 2029.32 & 1.21 & 2029.17 & 2029.17 & 0.00 \\
\hline
\end{tabular}

Table 1: OC and SC Frequencies w/ (right) and w/o (left) Electrodes BCs ; EMCC w/ and w/o Electrodes BCs

Equations 3, 4 can be transformed into the subspace spanned by the five first eigenmodes, see Thomas et al. [28]:

$$
\left\{\begin{array}{l}
\ddot{q}_{i}+2 \xi_{i} \omega_{0 i} \dot{q}_{i}+\omega_{0 i}^{2} q_{i}=K_{i} \omega_{0 i} \sqrt{C} V(t) \\
U(t)=\sum_{i=1}^{N} \Phi_{i} q_{i}(t)
\end{array}\right.
$$

where $C$ denotes the capacitance of the patches, and $V(t)$ denotes the excitation signal in Volt. Therefore the Frequency Response Function of the structure under electric excitation is given by:

$$
\frac{\hat{X}(j \omega)}{\hat{V}(j \omega)}=\sum_{i=1}^{N} \Phi_{i} \frac{\hat{q}_{i}(j \omega)}{\hat{V}(j \omega)}=\left(\sum_{i=1}^{N} \Phi_{i} \frac{K_{i} \omega_{0 i} \sqrt{C}}{-\omega^{2}+2 j \omega \xi_{i} \omega_{0 i}+\omega_{0 i}^{2}}\right)
$$

Fig. 4 shows the Frequency Response Function of the structure under electric excitation with piezoelectric patches located in the center of the beam on both sides with opposite polarization and a serial electric wiring. The sensor A is placed on the left of the 
beam and measure on $e_{y}$ direction, see Fig. 2. As expected, if the patches are wellmanufactured and well-glued, the response only show the first mode (blue curve). If there is a misalignment of $2 \mathrm{~mm}$ of the patches along direction $e_{x}$, the transfer function highlights a few coupling (red curve). And if the patches are not linked together, all the modes can be coupled (black curve). To conclude this section, let's say that a good design can allow to obtain a perfectly uncoupled excitation in order to follow only the first mode of the structure but as the perfection is not human, the robustness of the experiments can be improved by a good choice off excitation signal, see next section.
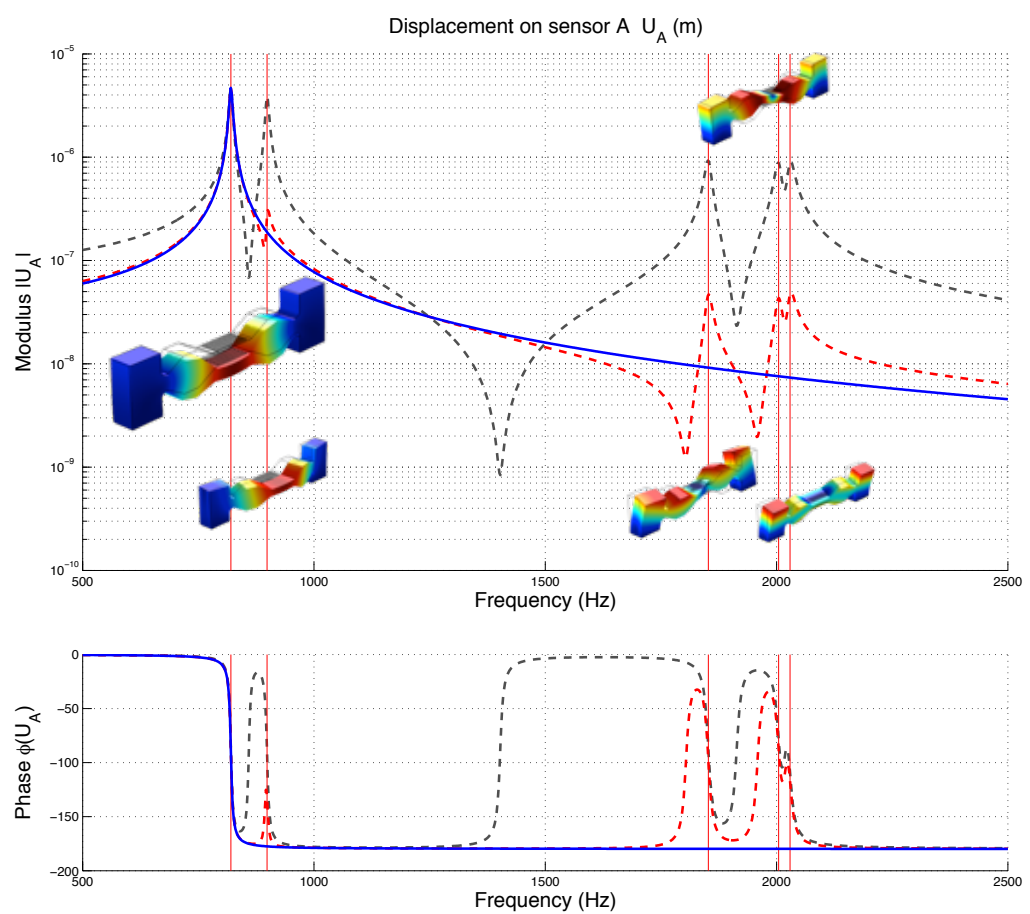

Figure 4: Frequency Response Function of the structure. Blue: perfectly manufactured specimen. Red: Misalignement of $2 \mathrm{~mm}$ of the patches. Black: maximum coupling when only a pair of patches is excited and the symmetries are broken. 


\section{Excitation Signal}

In order to get the dependence of the natural frequency and the modal damping to the amplitude of vibration, we assume that modal decomposition is frequency independent and we use a stop-sine excitation signal. The stop-sine signal is performed with tuned frequency targeted to the first natural frequency. The greatest difficulty is to produce an exact interruption of the excitation without introducing any perturbation and any transient response. In this section, we demonstrate that this technique allows to obtain a very little coupling between the modes. Moreover, we demonstrate that the interruption time is not of great importance. The dynamic model of the structure can be written in the modal base with this expression:

$$
\left\{\begin{array}{l}
\ddot{q}_{i}+2 \xi_{i}\left(q_{i}\right) \omega_{0 i}\left(q_{i}\right) \dot{q}_{i}+\omega_{0 i}\left(q_{i}\right)^{2} q_{i}=f_{i} \cos (\omega t) \\
U(t)=\sum_{i=1}^{N} \Phi_{i} q_{i}(t)
\end{array}\right.
$$

Considering the piezoelectric components for the excitation:

$$
f_{i}=K_{i} \omega_{0 i} \sqrt{C} V
$$

For steady state, assuming a first order Fourier Series Decomposition, modal solutions are sought of the form:

$$
\begin{aligned}
& q_{i}(t)=A_{i} \cos (\omega t)+B_{i} \sin (\omega t) \\
& \dot{q}_{i}(t)=-A_{i} \omega \sin (\omega t)+B_{i} \omega \cos (\omega t) \\
& \ddot{q}_{i}(t)=-A_{i} \omega^{2} \cos (\omega t)-B_{i} \omega^{2} \sin (\omega t)
\end{aligned}
$$

Using this solution form in equation 8:

$\left(A_{i} \omega_{0 i}^{2}+2 \xi_{i} \omega_{0 i} \omega B_{i}-A_{i} \omega^{2}\right) \cos (\omega t)+\left(\omega_{0 i}^{2} B_{i}-2 \xi_{i} \omega_{0 i} \omega A_{i}-B_{i} \omega^{2}\right) \sin (\omega t)=f_{i} \cos (\omega t)$

Thus, harmonic balance, between each Fourier term leads to:

$$
\left\{\begin{aligned}
B_{i} & =f_{i} \frac{2 \xi_{i} \omega_{0 i} \omega}{\left(\omega_{0 i}^{2}-\omega^{2}\right)^{2}+4 \xi_{i}^{2} \omega_{0 i}^{2} \omega^{2}} \\
A_{i} & =f_{i} \frac{\left(\omega_{0 i}^{2}-\omega^{2}\right)}{\left(\omega_{0 i}^{2}-\omega^{2}\right)^{2}+4 \xi_{i}^{2} \omega_{0 i}^{2} \omega^{2}}
\end{aligned}\right.
$$

The solution can be built using Modal Decomposition:

$$
U_{p}(t)=\sum_{i=1}^{N} \Phi_{i} q_{i}(t)
$$


This leads to:

$$
\begin{aligned}
U_{p}(t)= & \left(\sum_{i=1}^{N} \frac{\Phi_{i} f_{i}\left(\omega_{0 i}^{2}-\omega^{2}\right)}{\left(\omega_{0 i}^{2}-\omega^{2}\right)^{2}+4 \xi_{i}^{2} \omega_{0 i}^{2} \omega^{2}}\right) \cos (\omega t) \\
& +\left(\sum_{i=1}^{N} \frac{2 \Phi_{i} f_{i} \xi_{i} \omega_{0 i} \omega}{\left(\omega_{0 i}^{2}-\omega^{2}\right)^{2}+4 \xi_{i}^{2} \omega_{0 i}^{2} \omega^{2}}\right) \sin (\omega t)
\end{aligned}
$$

We define:

$$
C_{i}=\left(\omega_{0 i}^{2}-\omega^{2}\right)^{2}+4 \xi_{i}^{2} \omega_{0 i}^{2} \omega^{2}
$$

Thus Equation 14 becomes:

$$
\begin{array}{r}
U_{p}(t)=\sum_{i=1}^{N}\left(\frac{\Phi_{i} f_{i}\left(\omega_{0 i}^{2}-\omega^{2}\right) \prod_{k=1}^{N(k \neq i)} C_{k}}{\prod_{k=1}^{N} C_{k}}\right) \cos (\omega t)+ \\
\sum_{i=1}^{N}\left(\frac{2 \Phi_{i} f_{i} \xi_{i} \omega_{0 i} \omega \prod_{k=1}^{N(k \neq i)} C_{k}}{\prod_{k=1}^{N} C_{k}}\right) \sin (\omega t)
\end{array}
$$

Let us recall that one expects to get the maximum of colinearity with the first mode in order to track its parameters $\left(\xi_{1}\right.$ and $\left.\omega_{01}\right)$ evolution according to its instantaneous amplitude $q_{1}(t) . U_{p}(t)$ is fully collinear to the first mode $\Phi_{1}$ if the commands $f_{i}$ ( $i=$ $2 \ldots N)$ of the other modes are nil. As it is not in most of the cases, even if we placed the piezos in order to achieve this goal, it is possible to reach the maximum of colinearity with the first mode when $C_{1}$ is minimum, namely:

$$
\frac{\partial C_{1}}{\partial \omega}=-4 \omega\left(\omega_{01}^{2}-\omega^{2}\right)+8 \xi_{1}^{2} \omega_{01}^{2} \omega=4 \omega\left(\omega^{2}-\omega_{01}^{2}+2 \xi_{1}^{2} \omega_{01}^{2}\right)=0
$$

i.e. when the structure is excited at resonance frequency:

$$
\omega_{R}=\omega_{01} \sqrt{1-2 \xi_{1}^{2}}
$$

After the excitation stops, at time $t_{c}$, the transient states $q_{i}(t)$ are given by the homogeneous equations:

$$
\ddot{q}_{i}+2 \xi_{i} \omega_{0 i} \dot{q}_{i}+\omega_{0 i}^{2} q_{i}=0
$$


For each states, one reaches a solution of the form:

$$
\begin{aligned}
q_{i}(t)=\left(K_{i} \cos \left(\omega_{P i}\left(t-t_{c}\right)\right)+L_{i} \sin \left(\omega_{P i}\left(t-t_{c}\right)\right)\right) e^{-\xi_{i} \omega_{0 i}\left(t-t_{c}\right)} \\
\dot{q}_{i}(t)=-\xi_{i} \omega_{0 i}\left(K_{i} \cos \left(\omega_{P}\left(t-t_{c}\right)\right)+L_{i} \sin \left(\omega_{P i}\left(t-t_{c}\right)\right)\right) e^{-\xi_{i} \omega_{0 i}\left(t-t_{c}\right)}+ \\
\omega_{P i}\left(-K_{i} \sin \left(\omega_{P i}\left(t-t_{c}\right)\right)+L_{i} \cos \left(\omega_{P i}\left(t-t_{c}\right)\right)\right) e^{-\xi_{i} \omega_{0 i}\left(t-t_{c}\right)}
\end{aligned}
$$

The excitation cutting time $t_{c}$ is chosen such that $\left(t-t_{c}\right)=\frac{2 \mathrm{k} \pi}{\omega_{P}}$ with $k \in \mathbb{N}$ :

$$
\begin{aligned}
K_{i} & =q_{i}\left(\mathrm{t}_{\mathrm{c}}\right) \\
L_{i} & =\frac{-\xi_{i} \omega_{0 i} q_{i}\left(\mathrm{t}_{\mathrm{c}}\right)-\dot{q}_{i}\left(\mathrm{t}_{\mathrm{c}}\right)}{\omega_{P i}}
\end{aligned}
$$

At $t_{c}$, the steady state gives the following initial conditions for the transient states:

$$
\left\{\begin{array}{l}
q_{i}\left(\mathrm{t}_{\mathrm{c}}\right)=A_{i} \cos \left(\omega \mathrm{t}_{\mathrm{c}}\right)+B_{i} \sin \left(\omega \mathrm{t}_{\mathrm{c}}\right) \\
\dot{q}_{i}\left(\mathrm{t}_{\mathrm{c}}\right)=-A_{i} \omega \sin \left(\omega \mathrm{t}_{\mathrm{c}}\right)+B_{i} \omega \cos \left(\omega \mathrm{t}_{\mathrm{c}}\right)
\end{array}\right.
$$

The transient response can be also built using Modal Decomposition:

$$
U_{t}(t)=\sum_{i=1}^{N} \Phi_{i}\left(K_{i} \cos \left(\omega_{P i}\left(t-t_{c}\right)\right)+L_{i} \sin \left(\omega_{P i}\left(t-t_{c}\right)\right)\right) e^{-\xi_{i} \omega_{0 i}\left(t-t_{c}\right)}
$$

Obviously, if $U_{p}(t)$ is collinear to the first mode, $q_{i}(t)$ and $\dot{q}_{i}(t)$ are quasi nil for $i=2 \ldots 5$. To achieve this goal, in the first simulations: $f_{1}$ is greater than $f_{i}, i=2 \ldots 5$. To illustrate the fact that the cutting time has no influence, Figure 5 shows the signal computed with previous equations taking into account two different cutting times. In the time-domain, Fig.5, one can see that the signals are every similar. This is confirmed by the phase diagram, see Fig. 6, where the signals are perfectly superimposed. This Figure also highlights the fact that the value of $t_{c}$ only leads to a phase offset.

If the signal $U_{P}(t)$ is not collinear to the first mode, the transient response, i.e. for $t>t_{c}$ may be modulated by other frequency components. The parameters that lead to this situation are the excitation frequency $\omega$ and the commands $f_{i}$. The influence of the command is shown on Fig. 7. On can see that if $f_{2}>>f_{1}$, it is impossible to get an unmodulated signal even if the excitation frequency is equal to the resonant frequency. Notice that the greater is the modal damping, the greater is the modal coupling.

The influence of the excitation frequency is shown on Fig. 8. On can see that if $\omega=1.2 \omega_{01} \sqrt{1-2 \xi_{1}^{2}}$, it is impossible to get an unmodulated signal even if $f_{1}$ is greater 


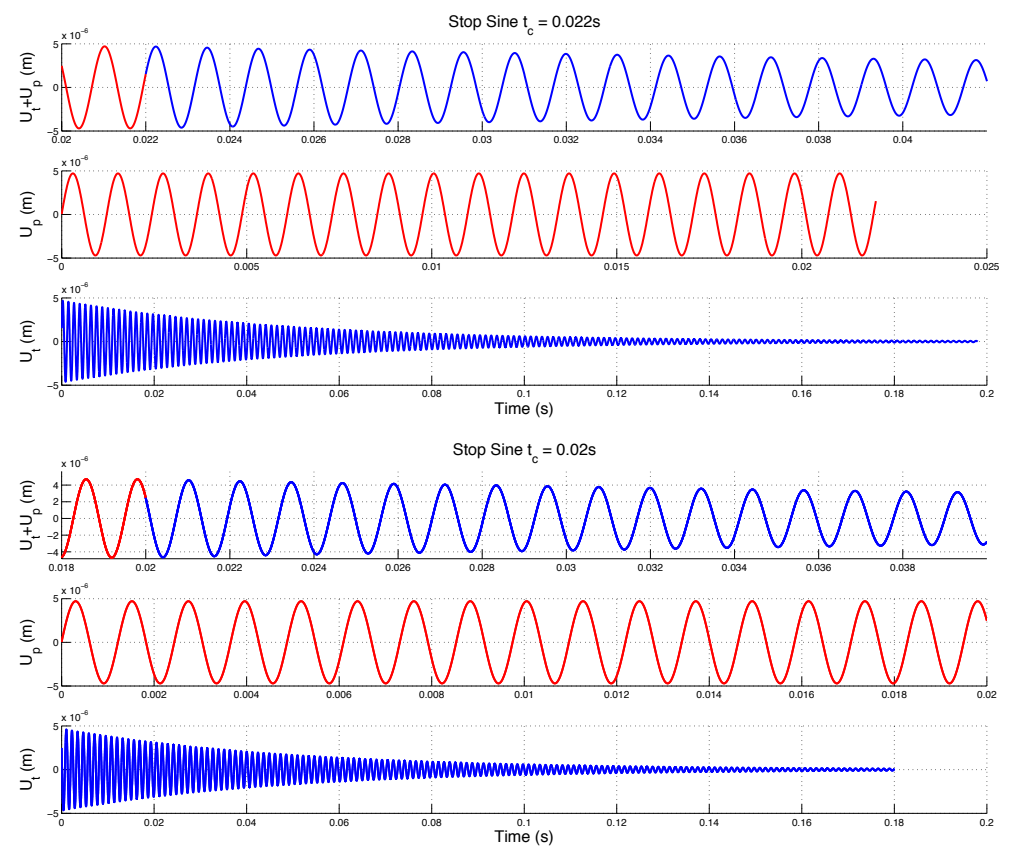

Figure 5: Stop Sine response for $t_{c}=0.022 \mathrm{~s}$ and $t_{c}=0.02 \mathrm{~s}$ 


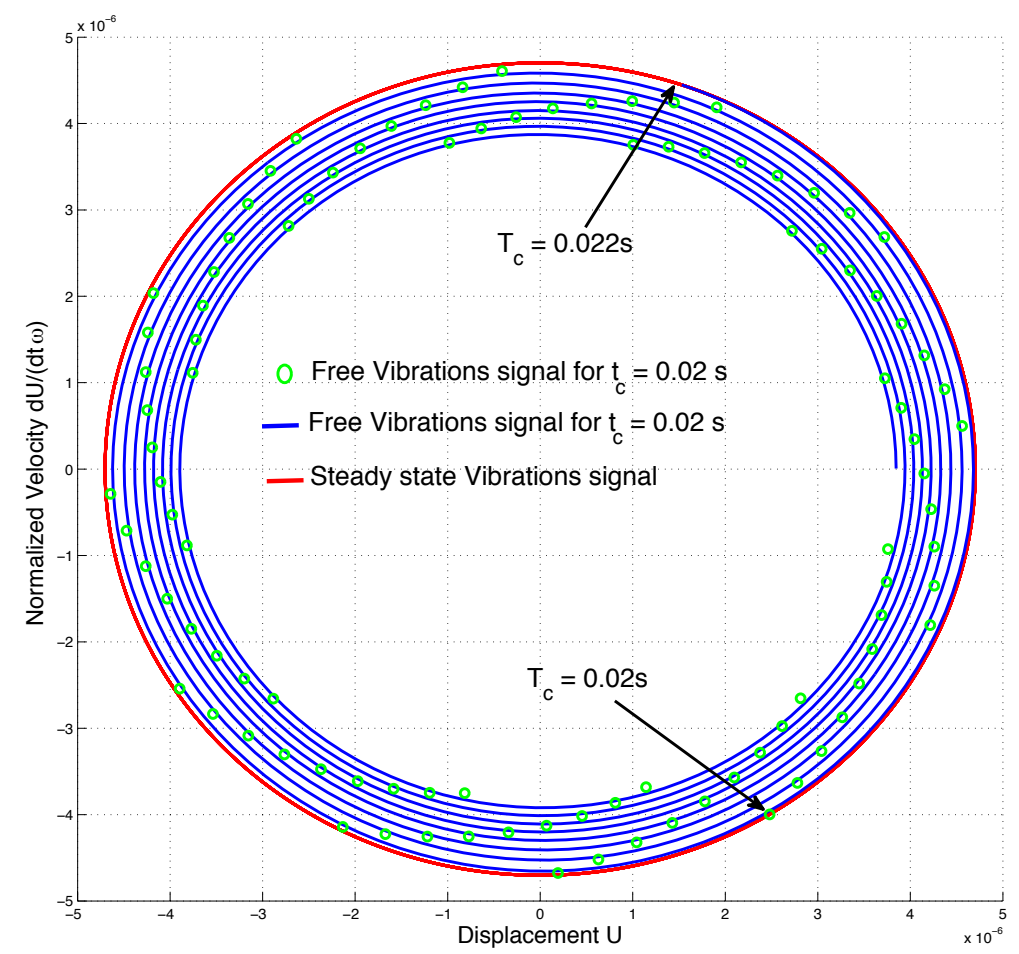

Figure 6: Phase Diagram of the responses for $t_{c}=0.022 \mathrm{~s}$ and $t_{c}=0.02 \mathrm{~s}$

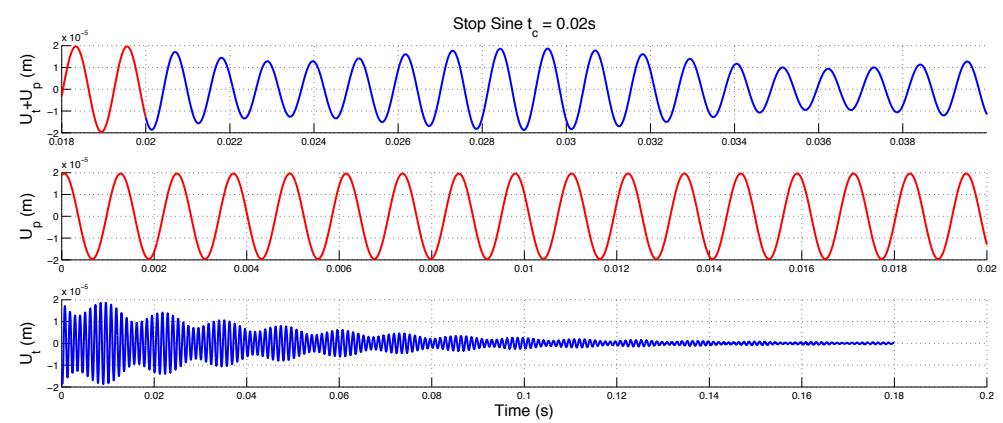

Figure 7: Stop Sine response for $f_{1}=1$ and $f_{2}=100$ and $\omega=\omega_{01} \sqrt{1-2 \xi_{1}^{2}}$ 

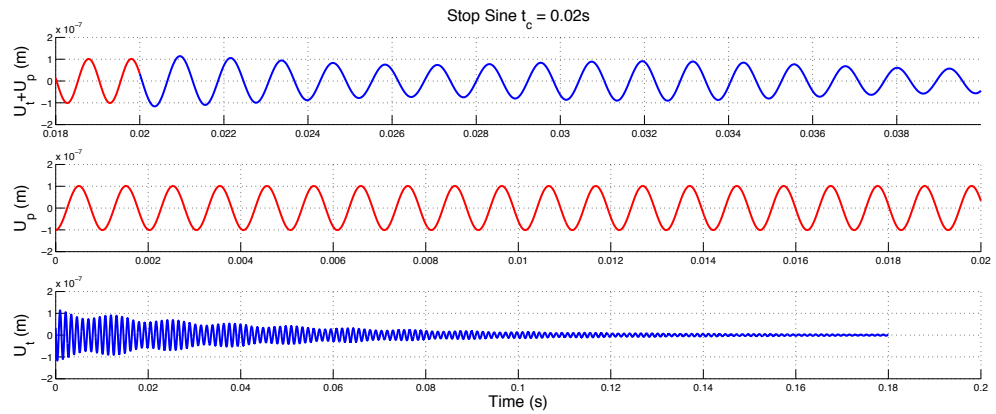

Figure 8: Stop Sine response for $f_{1}=1$ and $f_{2}=0.1$ and $\omega=1.2 \omega_{01} \sqrt{1-2 \xi_{1}^{2}}$

than $f_{2}$. To summarize, the influence of the $\omega$ and $f_{i}$ on the performance of stop-sine tests, the ratios $K_{1} / K_{2}$ and $L_{1} / L_{2}$ are plotted according to the ratios $\omega_{e} / \omega_{R}$ and $f_{1} / f_{2}$, see Fig. 9. From this figure, one can conclude that the excitation frequency $\omega_{e}$ might be in a narrow bandwidth around the resonance frequency $\omega_{R}$; And that $f_{2}$ has to be ten times lower than $f_{1}$ in order to get a good decoupling between the modes. This means that the previous study on the placement of piezos is of great interest in order to minimize the ratios $f_{i} / f_{1}$.

\section{Post-Processing using Kalman Filter}

\subsection{Tracking of damped sinusoidal components}

The filtering of damped sinusoidal components into a composite signal cannot be carried out by classical spectral analysis methods. ARMA filters [29] are not able to separate sinusoidal components and random noise into a same frequency bandwidth. Liftering techniques in cepstral analysis [30] and curve smoothing introduce important distortions in phase spectrum.

As instantaneous phases of modulated signals need to be accurately tracked, here a technique based on Extended Kalman filtering has been selected. Contrary to VoldKalman filtering, no frequency information is required. Different versions of statespace model have been proposed to model an amplitude and frequency modulated sine for robustness and stability reasons $[31,32,33,34,35]$. An appropriate state-space 

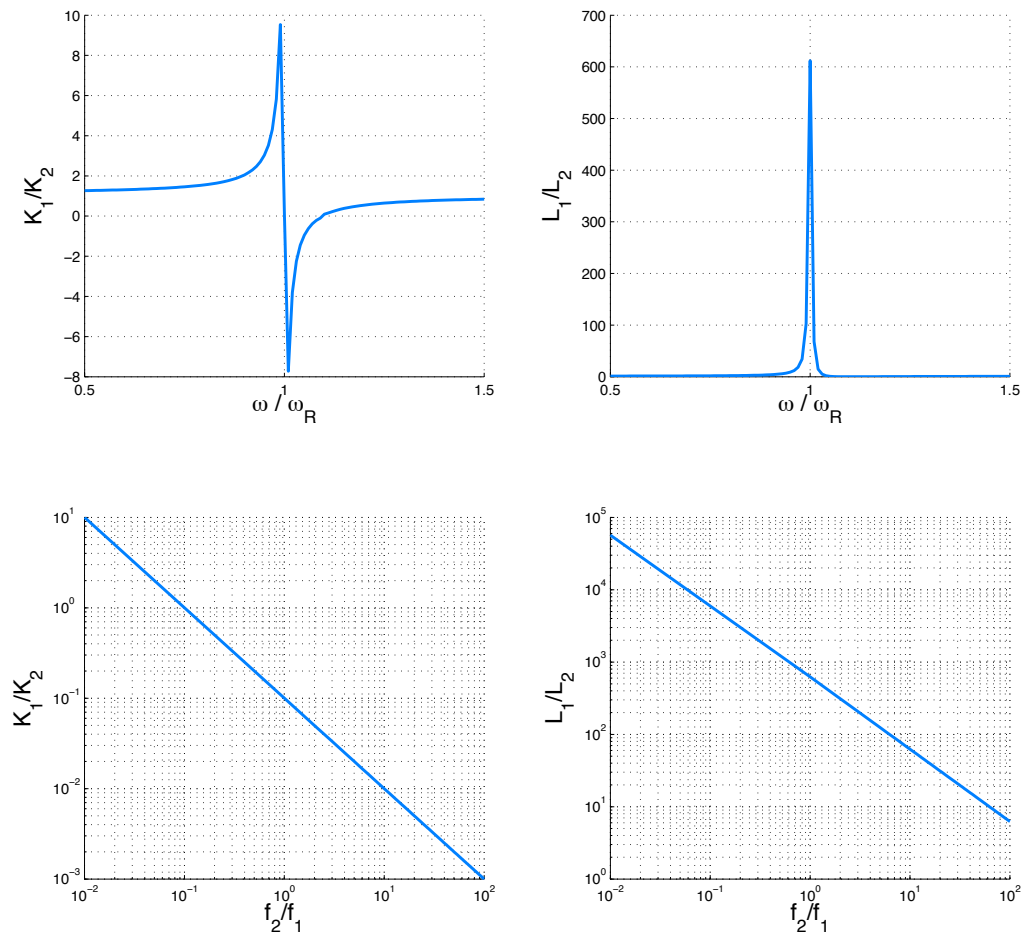

Figure 9: Excitation frequency $\omega$ and modal command $f_{2}$ influences on the ratios $K_{1} / K_{2}$ and $L_{1} / L_{2}$ 
model is chosen and detailed in the following paragraph, and formulas for parameterization are proposed.

4.2. Discrete state space formulation of an amplitude and frequency modulated sinusoid

The goal of this paragraph is to develop a state space formulation which is relevant on a short time scale. A sinusoid whose amplitude and frequency are modulated over time can be described in the complex domain as an analytic signal:

$$
x(t)=a(t) \exp (j \phi(t))
$$

where $a(t)$ is the instantaneous amplitude and $\phi(t)$ is the instantaneous phase. The discrete form of $x(t)$ at time step $t_{n}=n \Delta t$ is $x_{n}=x(n \Delta t)$. The complex variable $x_{n}$ can be divided into its real and imaginary parts $x_{n}=x_{1, n}+j x_{2, n}=q_{n}+j x_{2, n}$, with $q_{n}=x_{1, n}$ the modal displacement. A sinusoid that slightly varies over time can be approximated by:

$$
x_{n}=a_{n} \exp \left(j\left(2 \pi f_{n} n \Delta t\right)\right)
$$

where $f_{n}$ is the instantaneous frequency. As the parameters $f_{n}$ and $a_{n}$ of the sinusoid slightly vary over time, they are almost equal between two consecutive time steps. Then a transition formulation can be given from $x_{n}$ to $x_{n+1}$ :

$$
x_{n+1} \approx a_{n} \exp \left(j\left(2 \pi f_{n} n \Delta t\right)\right) \times \exp \left(j\left(2 \pi f_{n} \Delta t\right)\right)
$$

This approximation is only true if modulations of $a_{n}$ and $f_{n}$ are slower than the period of the sinusoid. This constraint is assumed to be verified. Then a linear transition is obtained between the imaginary part $x_{2}$ and the real part $x_{1}$, of $x_{n}$ and $x_{n+1}$ :

$$
\begin{aligned}
& x_{1, n+1}=x_{1, n} \cdot \cos \left(2 \pi f_{n} \Delta t\right)-x_{2, n} \cdot \sin \left(2 \pi f_{n} \Delta t\right) \\
& x_{2, n+1}=x_{1, n} \cdot \sin \left(2 \pi f_{n} \Delta t\right)+x_{2, n} \cdot \cos \left(2 \pi f_{n} \Delta t\right)
\end{aligned}
$$

or written in a matrix form

$$
\left(\begin{array}{c}
x_{1, n+1} \\
x_{2, n+1}
\end{array}\right)=\left[\begin{array}{cc}
\cos \left(2 \pi f_{n} \Delta t\right) & -\sin \left(2 \pi f_{n} \Delta t\right) \\
\sin \left(2 \pi f_{n} \Delta t\right) & \cos \left(2 \pi f_{n} \Delta t\right)
\end{array}\right]\left(\begin{array}{c}
x_{1, n} \\
x_{2, n}
\end{array}\right)
$$


The instantaneous amplitude $a_{n}$ is given by:

$$
a_{n}=\sqrt{x_{1, n}^{2}+x_{2, n}^{2}}
$$

As $a_{n}$ and $f_{n}$ should be allowed to vary over time, it is proposed here to use the following non linear state-space formulation:

$$
x_{n+1}=\Phi\left(X_{n}\right)+W_{n}
$$

where $X_{n}=\left(x_{1, n}, x_{2, n}, x_{3, n}\right)^{T}$ and $\mathrm{Wn}$ the process noise. A state variable $x_{3, n}=$ $2 \pi f_{n} \Delta t$ was added to track the evolution of the instantaneous frequency fn. The transition between two time steps is composed by a sum of two parts: the stationary part and the evolutionary part.

The stationary part links two successive points of a stationary sinusoid by $\Phi($.$) .$ Then $\Phi($.$) is assumed as the non-linear transition function and is given by \Phi\left(X_{n}\right)=$ $F_{n} X_{n}$, where :

$$
F_{n}=\left(\begin{array}{ccc}
\cos \left(x_{3, n}\right) & -\sin \left(x_{3, n}\right) & 0 \\
\sin \left(x_{3, n}\right) & \cos \left(x_{3, n}\right) & 0 \\
0 & 0 & 1
\end{array}\right)
$$

The two first components are related to the complex amplitude and are obtained by the previous linear relation. The third component $x_{3, n+1}=x_{3, n}$ constrains the frequency not to change strongly between two time steps. Up to now, the non-stationary behavior of the sinusoid was not modeled because it is not possible to formulate an exact equation for its evolution. We suppose that $W_{n}$ is a random variable whose probability law is Gaussian : $W_{n}=N(0, Q)$, where $Q$ is its variance matrix. Then variations of amplitude and frequency are allowed by random values of $W_{n}$.

In a first glance, it could seem strange to choose a random variable for an effect which is generally deterministic. For instance, the variation of frequency excitation due to an engine is mainly deterministic. Anyway, this state space does not need to represent accurately the evolution of a sinusoid on a long period, but only step by step. Then on a short time scale, a random evolution of an and $\mathrm{fn}$ is enough to model a non-stationary sinusoid. 
This state space formulation is nonlinear: the transition function $\Phi\left(X_{n}\right)$ is varying over time and depends on the frequency modulation.

In reality, only the real part $x_{1, n}$ of the analytic signal $X_{n}$ can be observed. Unlike the transition phase, the observation phase is completely linear

$$
\begin{aligned}
& U_{n}=\left[\begin{array}{lll}
1 & 0 & 0
\end{array}\right] X_{n}+V_{n} \\
& U_{n}=x_{1, n}+V_{n}
\end{aligned}
$$

where $V_{n}$ is a noise observation random process.

Finally, a nonlinear discrete state space model has been formulated to model the transition and observation of sinusoid components mixed with random processes

$$
\left\{\begin{array}{l}
X_{n+1}=\Phi\left(X_{n}\right)+W_{n} \\
U_{n+1}=H X_{n+1}+V_{n+1}
\end{array}\right.
$$

where $\phi($.$) is the nonlinear transition function given by \phi\left(X_{n}\right)=F\left(X_{n}\right) X_{n}$ and $H($. is the observation matrix given by:

$$
H=\left[\begin{array}{lll}
1 & 0 & 0
\end{array}\right]
$$

\subsection{Application to the Extended Kalman Filter}

Kalman filtering refers to a family of algorithms that track the temporal evolution of a dynamic model based on noised measurements:

$$
\begin{aligned}
X_{n+1} & =f\left(X_{n}, W_{n}\right) \\
U_{n+1} & =h\left(X_{n+1}, V_{n+1}\right)
\end{aligned}
$$

described here in discrete time domain. An efficient algorithm in terms of means and covariances can be derived when $f($.$) and h($.) are linear. Indeed it estimates the state probability distribution by its two first moments. Unfortunately, they are no longer sufficient to characterize the distribution in the nonlinear case. Then some approximations have to be done in order to find a practical algorithm.

The extended Kalman filtering is an adaptation of the classical Kalman filtering to problems with state dynamics governed by nonlinear transformations. Although it is 
not required here, it should be noticed that it can also handle a nonlinear transformation from state variables to measurement variables. It generally exhibits a good robustness because it uses linear approximation over small ranges of state space. Without any input control, the state model is given by the first equation in system eq. 33 where $W_{n}$ is the process noise assumed to be Gaussian $N(0, Q)$. The observation model is described by the second equation in system eq. 33 where $H X_{n+1}$ is the observation function and $V_{n+1}$ the observation noise assumed to be Gaussian $N(0, R)$. State and observation noises are assumed to be uncorrelated. The Extended Kalman Filter [36] is defined using predict and update phases. The predict phase gives an a priori estimate of the state and covariance based on previous time step $t_{n}$ :

Predicted state

$$
\hat{X}_{n+1 \mid n}=\hat{F}_{n \mid n} \hat{X}_{n \mid n}
$$

Predicted estimated covariance

$$
\hat{P}_{n+1 \mid n}=\tilde{F}_{n \mid n} \hat{P}_{n \mid n} \tilde{F}_{n \mid n}^{T}+Q
$$

And the update phase corrects the deviation of these estimations based on new observation at time step $t_{n+1}$ :

Innovation

$$
\tilde{Y}_{n+1 \mid n}=U_{n+1}-H \hat{X}_{n+1 \mid n}
$$

Innovation covariance

$$
S_{n+1}=H \hat{P}_{n+1 \mid n} H^{T}+R
$$

Kalman gain

$$
K g_{n+1}=\hat{P}_{n+1 \mid n} H^{T}\left(S_{n+1}\right)^{-1}
$$

Updated state estimate

$$
\hat{X}_{n+1 \mid n+1}=\hat{X}_{n+1 \mid n}+K g_{n+1} \tilde{Y}_{n+1 \mid n}
$$

Updated estimate covariance

$$
\hat{P}_{n+1 \mid n+1}=\left(I-K g_{n+1} H\right) \hat{P}_{n+1 \mid n}
$$


As the transition function $\phi$ is non-linear but differentiable, it is well locally approximated thanks to its Jacobian:

$$
\tilde{F}_{n \mid n}=\left.\left(\nabla_{X}(F(X) X)^{T}\right)^{T}\right|_{X=\hat{X}_{n \mid n}}
$$

In the case of $M$ modulated components, the first order derivative is required:

$$
\nabla X_{n}=\left(\begin{array}{ccccc}
\frac{\partial}{\partial x_{1, n}} & \frac{\partial}{\partial x_{2, n}} & \cdots & \frac{\partial}{\partial x_{3 M-1, n}} & \frac{\partial}{\partial x_{3 M, n}}
\end{array}\right)^{T}
$$

and the approximation becomes:

$$
\tilde{F}_{n, i}=\left[\begin{array}{ccc}
\cos \left(\hat{x}_{3, n}\right) & -\sin \left(\hat{x}_{3, n}\right) & -\hat{x}_{1, n} \sin \left(\hat{x}_{3, n}\right)-\hat{x}_{2, n} \cos \left(\hat{x}_{3, n}\right) \\
\sin \left(\hat{x}_{3, n}\right) & \cos \left(\hat{x}_{3 n}\right) & \hat{x}_{1, n} \cos \left(\hat{x}_{3, n}\right)-\hat{x}_{2, n} \sin \left(\hat{x}_{3, n}\right) \\
0 & 0 & 1
\end{array}\right]
$$

\subsection{Non linear damping and frequency identification with Extended Kalman Filter}

The most accurate excitation in the context of our study is a stop sine, tuned near the natural frequency. When the sine is stopped, the structure response is governed by the free vibrations motion. This motions is spatially colinear to the eigenmode studied (see figure 10). This property is significant and allows studying non linear dynamics with accuracy. Decreasing magnitude is used to study non-linear damping and backbone, by assuming that the damping depends on the displacement magnitude. The non linearity is assumed to be identify as an evolution of the natural frequency and damping of the first bending mode versus vibration amplitude.

In order to identify the instantaneous damping and frequency, three time-identifications methods are compared:

- The zero counting method consists in estimating the frequency with the time gap (number of samples) between two zero-crossing point of the time signal.

- The Hilbert method consists in the synthesis of the analytic signal (complex) composed with the original one and its Hilbert transform which composes the imaginary part. The complex signal allow to compute instanateous amplitude ans phase. The instantaneous frequency is the time derivative of the instanteous phase [10]. 


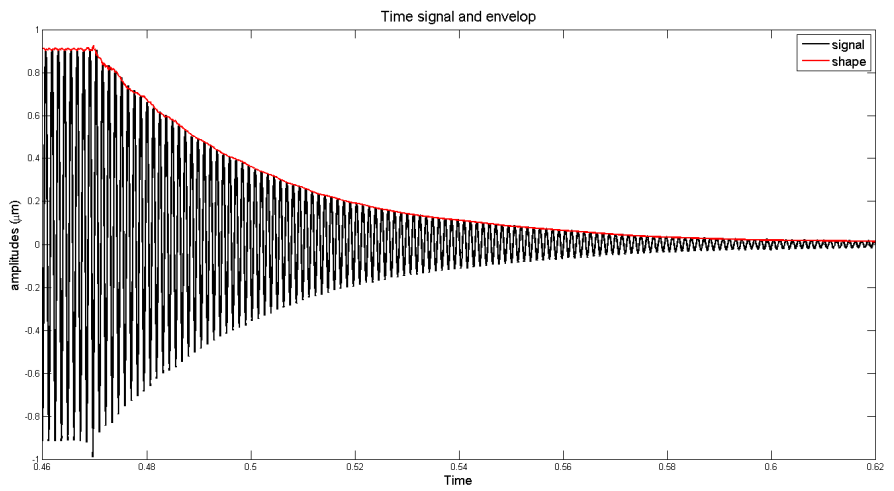

Figure 10: time signal and envelop of the tuned stop sine

- The Kalman Filter method [37] is used in order to avoid edge effects and oscilating envelop.

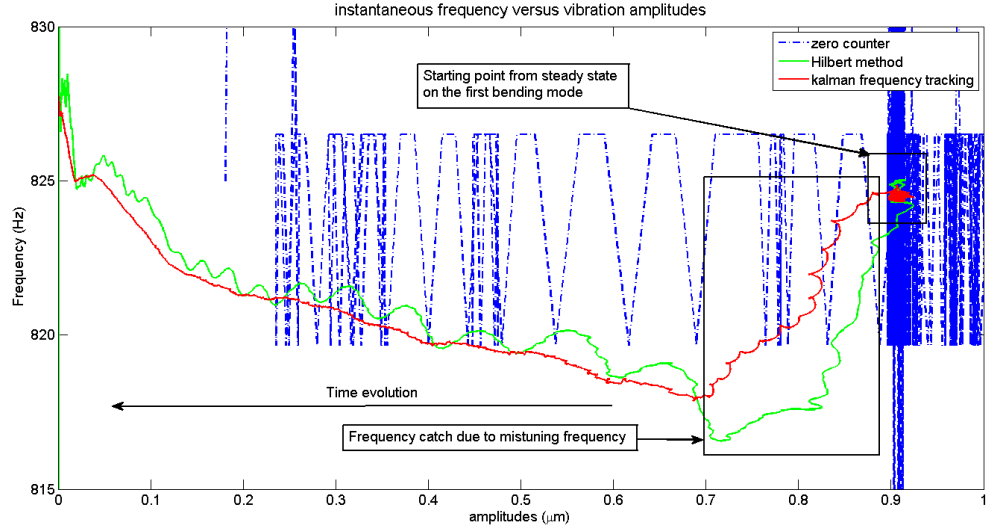

Figure 11: Evolution of the natural frequency of the first bending mode versus the displacement magnitude with three identification techniques.

Figure 11 and Figure 12 show the evolution of the natural frequency and the evolution of modal damping versus the displacement magnitude respectively. The curves are to be read from the right to the left, because the time-signal starts from large amplitudes and reaches null amplitudes, see Fig. 10. 


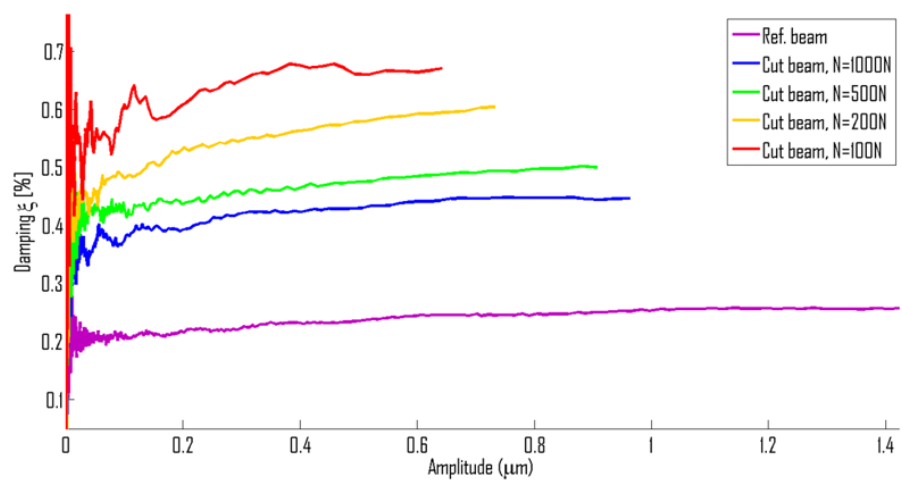

Figure 12: Evolutions of the damping versus the displacement magnitude for 4 different normal loads and a monolithic specimen

On Figure 11, the starting frequency is mistuned and the very first free oscillations cannot be used for non linear identification. During this short phase, the identification process shifts from the forced frequency to the natural frequency. The identification process starts from $0.7 \mu \mathrm{m}$ and ends at $0 \mu \mathrm{m}$. The natural frequency of the bending mode of our specimen is amplitude dependent. Friction in the interfaces acts as a softening effet.

The damping also depends on the displacement magnitude. Figure 12 represents the evolution of the first bending mode damping according to the displacement magnitude. The results obtained for the reference beam are compared with those obtained for the cut beam loaded with four different normal loads. These curves highlight that the damping depends on the displacement amplitude and on the normal load. As the normal load increases, the damping increases. This is due to the fact that there is less friction and partial sliding when the normal load is greater. The damping increases as the displacement magnitude increases. This is due to the augmentation of the sliding area according to a behavior very close to the one described by Mindlin [38] and more recently by Peyret et al. [22]. The damping evolution may be different after the vibration amplitude for which total sliding occurs, see Festjens et al. [7].

As both the damping and the natural frequency lead to the same exponential term that makes the vibration amplitude decrease, see eqn (20), one can wonder if the damp- 
ing increasing can be an artefact due to the frequency decreasing. Nevertheless, thanks to the signal decomposition we did, eqns (28) and (29), we get enough information to identify them separately and to be self confident in their own evolutions.

\section{Conclusions}

The goal of this study is to analyze transient vibrations in order to identify the relationship between the natural frequency, the modal damping and the vibration amplitude considering each mode separately. To achieve this goal, we propose a solution based on three key points : the load is applied by piezoelectric patches, the excitation signal is a stop-sine, the signal processing algorithm, for parameters identification, is based on the Extended Kalman filter.

In comparison with a shaker, the piezoelectric patches are embedded on the structure. Their mechanical behavior does not shift enough to modify the natural frequency of the structure when the electrical excitation is stopped. Moreover the patches are embedded on the structure on a position which provide a great electro-mechanical coupling for the desired mode and a very small coupling with the others.

In spite of the great advantages given by the patches and due to manufacturing errors, there is still a risk of coupling with others modes. To avoid the excitation of the others modes, we use the stop-sine to excite the structure at a frequency as close as possible to the resonance frequency. This allows to have stationary vibrations close to the desired mode. Thus, at the excitation cutting-time, the initial conditions of the free vibrations are colinear to the eigenmode we want to observe.

The processing of the signal by Kalman filtering allows to denoise the signal, even if it remains small coupling between the modes. Thanks to the measurements, the combined action of patches and "stop sines", the modal uncoupling is very efficient and the processing of the signal by the Hilbert Transform can also efficiently be used to obtain the parametric identification for instantaneous frequency and damping.

The main limitation of this process is the effective power of the piezoelectric patches that remains low especially for the excitation of massive and stiff structures. 


\section{References}

\section{References}

[1] M. Berthillier, C. Dupont, R. Mondal, Barrau, Blades forced response analysis with friction dampers., Transactions of the American Society of Mechanical Engineers 120 (1998) 468-474.

[2] O. Poudou, C. Pierre, Blades forced response analysis with friction dampers, Ph.D. thesis, University of Michigan (2007).

[3] L. Goodman, J. Klumpp, Analysis of slip damping with reference to turbine blade vibration, ASME Applied Mechanics Division 23 (3) (1956) 421-429.

[4] C. Beards, J. Williams, The damping of structural vibration by rotational slip in joints, Journal of Sound and Vibration 53 (3) (1977) 333-340.

[5] T. Pian, Structural damping of a simple built-up beam with riveted joints in bending, ASME Journal of Applied Mechanics 24 (1957) 35-38.

[6] E. Ungar, The status of engineering knowledge concerning the damping of builtup structures, Journal of Sound and Vibration 26 (1) (1973) 141-154.

[7] H. Festjens, G. Chevallier, J.-1. Dion, A numerical tool for the design of assembled structures under dynamic loads, International Journal of Mechanical Sciences 75 (2013) 170-177.

[8] A. Caignot, P. Ladeveze, D. Neron, J.-F. Durand, Virtual testing for the prediction of damping in joints, Engineering Computations 27 (5) (2010) 621-644.

[9] H. Festjens, G. Chevallier, J. Dion, Nonlinear model order reduction of jointed structures for dynamic analysis, Journal of Sound and Vibration 333 (7) (2014) $2100-2113$.

[10] J.-L. Dion, G. Chevallier, N. Peyret, Improvement of measurement techniques for damping induced by micro-sliding, Mechanical Systems and Signal Processing 34 (1-2) (2012) 106-115. 
[11] H. Ahmadian, H. Jalali, Identification of bolted lap joints parameters in assembled structures, Mechanical Systems and Signal Processing 21 (2) (2007) 1041-1050.

[12] A. F. Metherell, S. Diller, Instantaneous energy dissipation rate in a lap joint?uniform clamping pressure, Journal of Applied Mechanics 35 (1) (1968) $123-128$.

[13] J. Esteban, C. A. Rogers, Energy dissipation through joints: theory and experiments, Computers \& Structures 75 (4) (2000) 347-359.

[14] Y. Song, Simulation of dynamics of beam structures with bolted joints using adjusted iwan beam elements, Journal of Sound and Vibration 273 (1-2) (2004) 249-276.

[15] H. Goyder, P. Ind, D. Brown, Development of a method for measuring damping in bolted joints, in: Proc. IDTEC ASME, 2009.

[16] L. Heller, Damping in assemblies structures, Ph.D. thesis, Universite de FrancheComte (2005).

[17] L. Heller, E. Foltete, J. Piranda, Experimental identification of nonlinear dynamic properties of built-up structures, Journal of Sound and Vibration 327 (1) (2009) 183-196.

[18] B. Nanda, A. Behera, Study on damping in layered and jointed structures with uniform pressure distribution at the interfaces, Journal of Sound and Vibration 226 (4) (1999) 607-624.

[19] B. Nanda, Study of the effect of bolt diameter and washer on damping in layered and jointed structures, Journal of Sound and Vibration 290 (3-5) (2006) 12861314.

[20] L. Gaul, Friction control for vibration suppression, Mechanical Systems and Signal Processing 14 (2) (2000) 139-150. 
[21] H. Nouira, Experimental characterization and modeling of microsliding on a small cantilever quartz beam, Journal of Sound and Vibration 317 (1-2) (2008) $30-49$.

[22] N. Peyret, J.-L. Dion, G. Chevallier, P. Argoul, Micro-slip induced damping in planar contact under constant and uniform normal stress, International Journal of Applied Mechanics 02 (02) (2010) 281-304.

[23] Y. Ren, C. Beards, Identification of joint properties of a structure using frf data, Journal of Sound and Vibration 186 (4) (1995) 567-587.

[24] P. Argoul, T. Le, Instantaneous indicators of structural behaviour based on continuous cauchy wavelet transform, Mechanical Systems and Signal Processing 17 (2003) 243-250.

[25] A. Benjeddou, Advances in piezoelectric finite element modeling of adaptive structural elements: a survey, Computers \& Structures 76 (1) (2000) 347-363.

[26] G. Chevallier, S. Ghorbel, A. Benjeddou, A benchmark for free vibration and effective coupling of thick piezoelectric smart structures, Smart Materials and Structures 17 (6) (2008) 065007.

[27] G. Chevallier, S. Ghorbel, A. Benjeddou, Piezoceramic shunted damping concept: testing, modelling and correlation, Mécanique \& Industries 10 (05) (2009) 397411.

[28] O. Thomas, J.-F. Deü, J. Ducarne, Vibrations of an elastic structure with shunted piezoelectric patches: efficient finite element formulation and electromechanical coupling coefficients, International journal for numerical methods in engineering 80 (2) (2009) 235-268.

[29] T. Parks, C. Burrus, A new approach to linear filtering and prediction problems, Digital Filter Design, New York, John Wiley \& Sons Chapter 7.

[30] B. Randall, Cepstrum analysis, in: Encyclopedia of Vibration. Elsevier, Oxford 82 (2001) 216-227. 
[31] B. F. La Scala, B. R. Bitmead, M. R. James, Conditions for stability of the extended kalman filter and their application to the frequency tracking problem, Mathematics of Control, Signals, and Systems 8 (1995) 1-26.

[32] B. F. La Scala, B. R. Bitmead, Design of an extended kalman filter frequency tracker., IEEE Transactions on Signal Processing 44 (3) (1996) 739-742.

[33] B. F. La Scala, B. R. Bitmead, B. Quinn, An extended kalman filter frequency tracker for high-noise environments, IEEE Transactions on Signal Processing 44 (3) (1996) 431-434.

[34] S. Bittanti, S. Savaresi, On the parameterization and design of an extended kalman filter frequency tracker, IEEE Transactions on Automatic Control 45 (9) (2000) $1718-1724$.

[35] H. Hajimolahoseini, M. Reza Taban, H. Soltanian-Zadeh, Extended kalman filter frequency tracker for nonstationary harmonic signals, Measurement 45 (2012) $126-132$.

[36] R. E. Kalman, A new approach to linear filtering and prediction problems, Transactions of the American Society of Mechanical Engineers - Series D - Journal of Basic Engineering 82 (1960) 35-45.

[37] J.-L. Dion, C. Stephan, G. Chevallier, H. Festjens, Tracking and removing modulated sinusoidal components: A solution based on the kurtosis and the extended kalman filter, Mechanical Systems and Signal Processing 38 (2) (2013) 428 439.

[38] R. Mindlin, Compliance of elastic bodies in contact, ASME Journal of applied mechanics 16 (1949) 259-268.

\section{Appendix}

Appendix A: Piezoceramic manufacturer data

The piezoceramic manufacturer's useful data are the following. Mass density: $\rho=7800 \mathrm{~kg} \cdot \mathrm{m}^{-3}$ 
Elastic compliance: $s_{11}^{E}=16.1 \times 10^{-12} \mathrm{~m}^{2} \mathrm{~N}^{-1}, s_{33}^{E}=20.7 \times 10^{-12} \mathrm{~m}^{2} \mathrm{~N}^{-1}$

Strain piezoelectric constants: $d_{31}=-180 \times 10^{-12} C N^{-1}, d_{33}=400 \times 10^{-12} C N^{-1}, d_{15}=$ $550 \times 10^{-12} \mathrm{CN}^{-1}$

Relative dielectric constants: $\epsilon_{r 11}^{T}=1650, \in_{r 33}^{T}=1750\left(\rightarrow \epsilon_{11}^{T}=14.6 n \mathrm{Fm}^{-1}, \in_{33}^{T}=15.5 \mathrm{nFm}^{-1}\right)$

Material coupling factor: $k_{31}=0.35$

Appendix B: Specimen informations

The specimen is a machined, made of steel C35. The Young modulus of the material is equal to 220 GPa and its Poisson ratio is equal to 0,3.The dimensions are summarized on Figure 13.
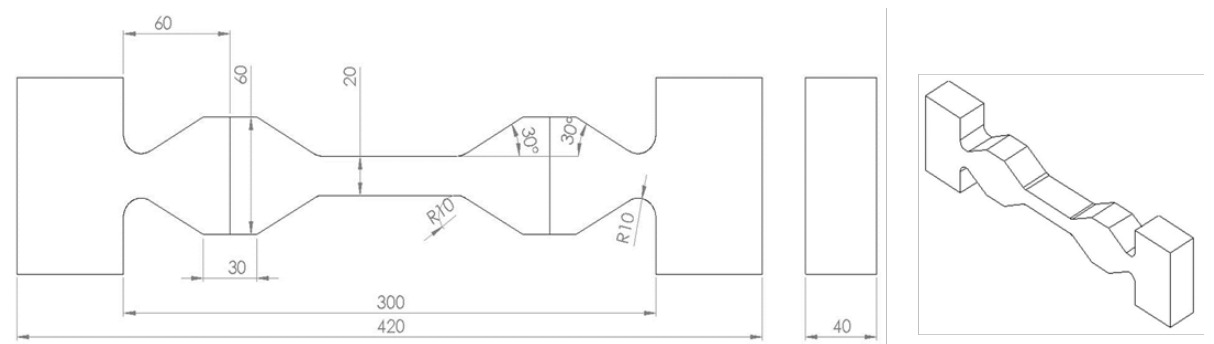

Figure 13: Geometry of the specimen.

Appendix C: Acronyms

EKF Extended Kalman Filter

EMCC Electro-Mechanical Coupling Coefficient

EL Energy Losses

FRF Frequency Response Functions

BC Boundary Conditions

SC Short Circuit

OC Open Circuit

FE Finite Element 


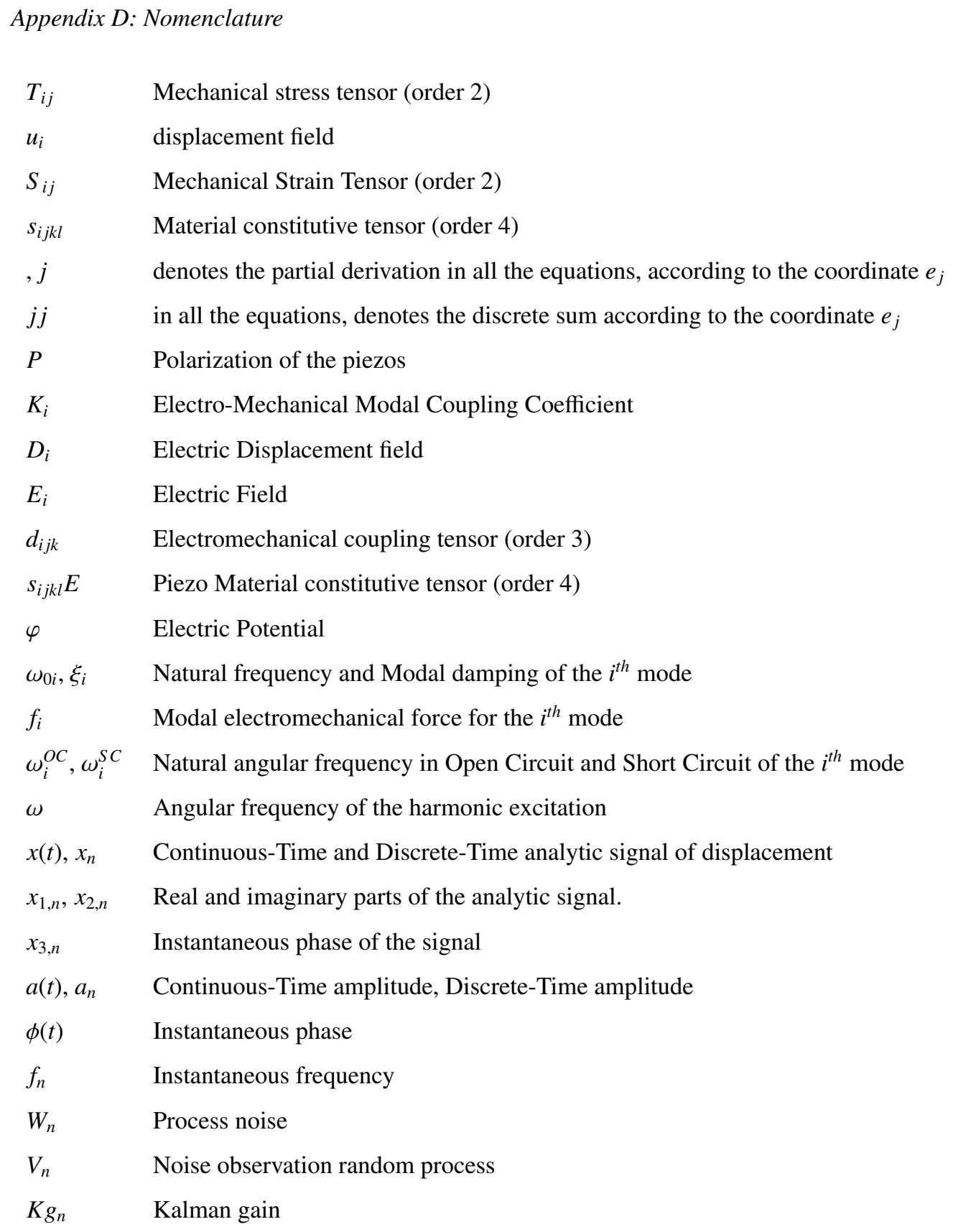

